\section{Session 23. Reproductive endocrinology}

130. Long term effects of time, pregnancy and medical treatment on plasma prolactin levels in 176 hyperprolactinemic women

A.M. Mattei, V. Cavioni, G. testa, A Zaro, and P.G. Crosignani III Department of Obstetrics and Gynaecology, University of Milano, Italy

In order to show evidence of prolactin changes specifically related to drug use, pregnancy or the simple effect of time, the evolution of plasma prolactin (PRL) levels were studied in 176 hyperprolactinemic women (87 carrying a prolactinoma) over a mean period of four years (range between 6-180 months).

Clinical presentation of the patients was amenorrhea for 110 , oligomenorrhea for 37 while 29 had normal but anovulatory cycles.

107 patients underwent 191 cycles of medical tretament (mean length 11 months; range 2-43), 73 got pregnant spontaneously or during drug intake while in 38 subjects spontaneous evolution of plasma prolactin concentration was evaluated over a mean period of 19 months (range 6-72).

Evaluation of prolactin changes after medical treatment pregnancy or just waiting periods showed a significant lower mean PRL level only in the idiopathic group of patients after pregnancy.

PRL trend of the individual values in both groups of patients indicate that: pregnancy normalizes prolactin concentration in $49 \%$ of patients and medical therapy is linked with a normalization rate of $27 \%$.

131. Dose-dependent suppression of serum Prolactin by cabergoline in hyperprolactinemic patients: a placebo controlled, randomized, double blind multicentre study

J. Webster ${ }^{1}$, M.F. Scalon ${ }^{1}$, A. D'Alberton, L. Falsetti, C. Ferrari, P. Fioretti, G. Giordano, M. L'Hermite, F. Camanni, P.G. Crosignani, L. De Cecco, R. Fadini, G. Faglia, F. Flamigni, and G. Tamburrano

European Multicentre Study Group; ${ }^{1}$ Neuroendocrine Unit, Department of Medicine, University of Wales, College of Medicine, Cardiff, Wales, United Kingdom

Cabergoline is a novel dopamine agonist with very longlasting (7-14 days) Prolactin (PRL)-lowering activity after oral administration. We have investigated the efficacy and tolerability of a range of doses of cabergoline in suppressing Prolactin in hyperprolactanemic women.

Patients and methods. This was a multi-centre, prospective, randomized, double-blind study involving 188 women with hyperprolactinemia secondary to a microprolactinoma $(n=113$, $60.1 \%)$, ideopathic disease $(n=67,35.6 \%)$, empty sella syndrome $(n=5,2.7 \%)$, or following failed surgery for prolactinoma $(n=3$, $1.6 \%$ ). The mean age was 31.8 years (range 16-46). Patients received either placebo $(n=20)$ or cabergoline $0.125 \mathrm{mg}(n=43)$, $0.5 \mathrm{mg}(\mathrm{n}=42), 0.75 \mathrm{mg}(\mathrm{n}=42)$ or $1 \mathrm{mg}(\mathrm{n}=41)$ twice weekly for four weeks, the first week's treatment being half dose; There were no significant differences in age, diagnosis $r$ previous therapy between the 5 treatment groups; mean ( \pm SEM) pre-treatment prolactins were $65.0 \pm 6.3,90.8 \pm 14.8,92.7 \pm 13.8,129.0 \pm$ 20.1 and $70.0 \pm 6.6 \mathrm{ng}: \mathrm{ml}$ respectively.

Results; After 4 weeks treatment PRL was suppressed to below half the pre-treatment level in $5 \%, 60 \%, 90 \%, 95 \%$ and $98 \%$, and completely normalized in $0 \%, 30 \%, 74 \%$, and $95 \%$ of patients taking placebo or cabergoline $(0.125 \mathrm{mg}, 0.5 \mathrm{mg}, 0.75$ $\mathrm{mg}$ and $1 \mathrm{mg}$ twice weekly respectively, showing a dose-response relationship among cabergoline doses (Armitage test: $p<<0.01$ ). In contrast, at these doses, adverse effects were reported in $45 \%$, $44 \%, 50 \%$ and $58 \%$ of patients with no evident dose relationship. Over $95 \%$ of reported side effects were relatively trivial, most frequently transient nausea, headache and dizziness. Severe adverse events including dizziness, nausea, headache and fatigue occurred in 13 patients taking cabergoline $(7.7 \%)$ but treatment had to be discontinued in only one patient who experienced severe dizziness.

Conclusions. Cabergoline is effective in normalizing PRL in hyperprolactinemia at doses of $0.5-1.0 \mathrm{mg}$ twice weekly. Its tolerability and simple administration schedule represent a significant therapeutic advance in the management of hyperprolactinemia.

132. Parlodel LAR in the chronic treatment of hyperprolactinaemic patients

A. Pedroncelli, M. Montini, G. Pagani, M.D. Pagani, D. Gianola, F. Tengattini, L. Cortesi, F. Gherardi, F. Sileo, and I. Lancranjan 1

Department of Endocrinology, Neuroendocrine Unit, United Hospitals, Bergamo, Italy, and ${ }^{1}$ Department of Neuroendocrinology, Clinical Research, Sandoz Pharma Ltd, Basel, Switzerland

Parlodel LAR, a new injectable long-acting form of bromocriptine, was administered to 78 patients (63 women and 15 men) affected by prolactinomas. At the CT-Scan/NMR macroadenoma was present in $31 / 78$ patients (pts). The initial dose of Pardlodel LAR was $25 \mathrm{mg}$ in $8 \mathrm{pts}, 50 \mathrm{mg}$ in 62 and $100 \mathrm{mg}$ in the other $8 \mathrm{pts}$. All pts received repeated injections of Parlodel LAR at the dose of $25-200 \mathrm{mg}$ every 28-60 days for 3-48 months. In every patient clinical evaluation, PRL plasma levels, tumor size evaluation and visual field (VF) examination were monitored. A marked decrease in PRL plasma levels was achieved in all patients whereas PRL normalization in $45 / 78$ pts after the first injection of Parlodel LAR. Eighteen of 78 pts also had bromocriptin plasma levels assessed after the first injection of Parlodel LAR $25 \mathrm{mg}, 50 \mathrm{mg}$ or $100 \mathrm{mg}$ (6 pts/dose) to assess the dose proportionality of this injectable bromocriptin preparation. Fifteen of 18 pts ,ormalized PRL plasma levels independently of the dose. The duration of PRL suppression/normalization lasted 7 to 60 days with a positive correlation between the length of efficacy and the initial dose used. At the CT-Scan/NMR a marked tumor shrinkage (20-60\%) was observed in 19/31 pts with macroprolactinoma within the first 28 days after the first dose of Parlodel LAR 50mg. After 6-12 month's therapy complete disappearance of the pituitary tumor was documented in $3 / 31$ pts. Three of the 4 pts who had VF defects obtained a complete and quick normalization of their VF within 12-72 hours after the first injection of Parlodel LAR. The clinical symptoms/signs recorded in 72/78 pts regressed in 69/72 pts after 14-700 days treatment. No important side effects were reported except in 2 cases. Patients' compliance was very good due to the therapeutic 
efficacy, good tolerability and long-lasting action of Parlodel LAR. Therefore, in conclusion, Parlodel LAR could be considered the first choice treatment in pts with prolactinoma.

133. Menses alteration in relationship to various precentage of weight loss

E. Pasinetti, L. Falsetti, and A. Gastaldi

Department of Obstetrics and Gynaecology, University of Brescia, Italy

We have evaluated the clinical and hormonal profile 203 of women, aged 18 to 26 years, who in hte last year had suffered a loss of wieght of $10-14 \%$ (group A), of $15-20 \%$ (group B) and more $20 \%$ (group C) in comparison with their intial weight. The frequency of amenorrhea and regular cycles was found to be greatly influenced by weight loss: in group A we saw amenorrhea in $75.3 \%$ of cases and regular cycles in $24.7 \%$; in group B we saw amenorrhea in $87.2 \%$ of cases and regular cycles in $12.8 \%$; in group $\mathrm{C}$ amenorrhea was present in $95.2 \%$ of cases and regular cycles in $4.8 \%$.

Amonst those women with regular menses, all included in groups $\mathrm{A}$ and $\mathrm{B}$, there was a major incidence of luteal phase defect (34.8\% in $A ; 16.7 \%$ in $B$ ) showing variations in the secretion of gonadotropin-realising hormone $(\mathrm{GnRH})$ and in the luteinizing hormone (LH) peak, caused by the former.

The presence of a certain percentage of regular cycles including those in groups with a greater weight loss, demonstrate various responses to stress.

The endocrinal evaluation showed in amenorrheic women a gradual decrease in the blood levels of gonadotrophin and estradiol, in groups A and C. In groups A and B, eventhough we can note apparently normal plasmatic levels of luteinizing hormone, the hypoestrogenism and the high percentage of amenorrhea confirm that in cases of minor weight loss there exists a modification in the pulsatile secretion of $\mathrm{GnRH}$ and gonadotropin. In addition the mean plasma cortisol levels were significantly increased whereas the prolactin levels were lower than controls.

134. Effectiveness of urinary $L H$ rapid assay in infertile women treated with clomiphene citrate

M. Bertazzoli Grabinski Broglio, S. Simionato, M. Lucianetti, A. Miragoli

Clinica Ostetrica Ginecologica II, Università di Milano, Milan, Italy

Thirteen infertile patients (ages 25 - 40) previously submitted to Clomiphene Citrate (CC) therapy unsuccessfully, were treated again by $\mathrm{CC}$ at the same dose during 17 monitored cycles. Eight patients were affected by primary infertility while 3 had undergone a spontaneous abortion early in the pregnancy and 2 had a history of recurrent abortion. All patients had been evaluated by hysterosalpingography and/or laparoscopy without evidence of any abnormality. All CC cycles were controlled by intensive hormonal and ultrasound monitoring. When the dominant follicle diameter reached $14 \mathrm{~mm}$. urinary $\mathrm{LH}$ rapid assay was effected every twelve hours (at 8 am and $8 \mathrm{pm}$ ) up to ovulatory surge evidence. Five patients underwent uneventful pregnancy while 2 did not conceive probably because husbands' semen analyses showed severe oligo-asthenospermia.
The use of an urinary $\mathrm{LH}$ rapid assay seems effective in raising the pregnancy rate of $\mathrm{CC}$ therapy in infertile patients.

135. Cumulative pregnancy rates after LHRHtreatment in hypothalamic amenorrhea

D.D.M. Braat and J. Schoemaker

Academic Hospital of the "Vrije Universiteit", Amsterdam, The Netherlands

In 49 patients with hypothalamic amenorrhea 272 LHRH induced cycles were studied retrospectively. Pulse dose varied from 2 $100 \mu \mathrm{g} /$ pulse; most patients received 10 or $20 \mu \mathrm{g}$. No difference in ovulation and conception rate was found between 10 and 20 ugram pulse dose cycles. Cumulative pregnancy rates (CPR) were calculated using the life table method. Forty-eight patients had 244 ovulatory cycles of which 55 resulted in pregnancy; the CPR was $78 \%$ after 6 and $93 \%$ after 12 ovulatory cycles. Eight pregnancies $(=14.5 \%)$ ended in abortion, 5 pregnancies were multiple ( 4 twins, 1 triplet).

Subdivisions of patients were made with respect to the presence of additional infertility factors, history of weight-loss, actual weight, estrogenic status and primary versus secondary amenorrhea. Evaluation of only first treatments per patients revealed a significant higher CPR in patients without other infertility factors compared to those with other factors $(84 \%$ versus $60 \%$ after 6 cycles) $(p<0.05)$. No differences were found in life table curves of the other subdivisions.

Using life-table statistics our data confirm the general opinion that LHRH therapy is highly successful in patients with hypothalamic amenorrhea, especially if no other infertility factors are present.

136. Clincal study of a new triphasic oral contraceptive (SH D $415 \mathrm{G}$, Milvane) in insulin dependent diabetic patients

A. Pedroncelli, N. Mazzocchi, F. Tengattini, G. Lepore, L. Cortesi, M. Montini, D. Gianola, M.D. Pagani, and G. Pagani Department of Endorcinology, Diabetes Unit, United Hospitals, Bergamo, Italy

A new triphasic oral contraceptive (SH D 415 G, Milvane) was studied in 14 insulin-dependent diabetic (IDDM) women, aged $27-39$. IDDM was diagnosed 1.5-19 years ago. All the patients were on monocomponent human insulin adminstered twice a day in the form of preformed mixture (Actraphane $(B)$ ) at a variable dose of $26-52 \mathrm{U} / \mathrm{day}$. No patients presented with overweight higher than $10 \%$ of the ideal body weight (IBW). The metabolic parameters indicating glyco-metabolic balance (daily blood glucose curve, HbA1C, frutosamine, lipid profile, plasma uric acid), were within the normal range. Micro-albuminuria was recorded in 6/14 women, whereas glycosuria in 11/14 women and the latter was below $20 \mathrm{~g} / 24$ hours. All the patients were put on Milvane due to anticonceptional purposes and they were treated for at least 6 months without discontinuing the drug because of poor tolerance. Ovulation which was recorded in every patient was completely inhibited. The daily insulin requirement increased in all patients (from 4 to $8 \mathrm{U} /$ day) without statistical significantly change in blood glucose profile, $\mathrm{HbAlC}$ and body weight. In the past $7 / 14$ women had to discontinue other oral contraceptives due to the worsening of the glycometabolic 
balance. On the contrary, all the patients are still on Milvane. In conclusion, the anticonceptyional treatment with Milvane is not hithetto resulted to be able to worsen the metabolic balance in diabetic patients. Further studies are required during more prolonged treatment to draw any definitive conclusions.

137. Predictive value of the active renin assay for the early diagnosis of ectopic pregnancy

L. Cedard, Th.M. Mignot, K. Meunier, A. Maria ${ }^{1}$, A Guichard, P. Boyer, J.R. Zorn

U. 166 INSERM and Maternité Baudelocque, Paris; ${ }^{1}$ Hôpital Villeneuve-St-Georges, France

The increasing frequency of ectopic pregnancy and the need of its early diagnosis have focused our interest on the research of biochemical markers. We have previously studied the profile of hCG, progesterone, and renin in fertile cycles after IVF-ET or GIFT and shown that his last parameter did not increase in ectopic pregnancies contrary to evolutive or abortive intrauterine pregnancies. After having established the hormonal value in the plasma of 99 spontaneous ongoing pregnancies between the 4th and 10th week of amenorrhea we have compared them to the values observed in 21 ectopic and 20 abortive pregnancies. The hCG levels are significantly higher in ongoing pregnancies but there is no statistically significant difference between abortive and ectopic gestation. The progesterone levels are significantly lower in ectopic pregnancies than in intrauterine gestation but are not really discriminant. The best parameter appears to be low active renin immunoradiometric assay (Pasteur Diagnostic). The difference between ectopic pregnancy and evolutive or abortive intrauterine gestations is highly significant, the highest value corresponding to the 25 th pecentile of normal values. The association of low hCG $(<15000 \mathrm{UI} / \mathrm{ml})$ and low active renin $(\leq$ $30 \mathrm{ng} / \mathrm{ml}$ ) seems to be highly predictive of the ectopic pregnancy. The renin angiotensin system has an important role in the process of implantation, but an early signal could also be transmitted by the implanted embryo to the corpus luteum.

\section{Session 24. Reproductive endocrinology}

138. The effect of polychlorinated Biphenyls (PCB) on estradiol synthesis of human cumulus cells

K. van der Ven, H. van der Ven, A. Thiebold, S. Al-Hasani, U. Wagner, K. Diedrich, and D. Krebs

Department of Obstetrics and Gynaecology, University of Bonn, Bonn, Fed. Rep. of Germany

Chlorinated hydrocarbons, E.G. PCBs, DDT, HCB, can be found in various body tissues and fluids. In human follicular fluid $(n=10)$ we could determine the following concentrations of various PCB congeners (Ballschmitter $n^{\circ} 15,118,138,153,169$, 180) on estradiol synthesis of human cumulus cells was investigated. Cumulus cells were collected in an IVF programme and cultured in a modified Ham's F10 medium supplemented with $5 \%$ fetal calf serum and $50 \mathrm{ng} / \mathrm{ml} 17 \beta$ testosterone (cell concentration: $25000 / \mathrm{ml}$. The PCB congeners were added in concentrations between 0.1 and $1000 \mathrm{ng} / \mathrm{ml}$. The concentration of estradiol was determined in the cell free supernatants after a 24 hour culture period. All PCB congeners induced a dose dependent reduction of estradiol synthesis by human cumulus cells. The various PCB congeners showed different biological activities in regard to the inhibitory effect on estradiol secretion. A decrease of estradiol secretion could already be observed in PCB concentrations that correspond to the average load of human follicular fluid.

139. Endocrine and immunological chances in women with chronic wood preservative exposition

I. Gerhard, M. Derner, B. Runnebaum

Department of Gynaecological Endocrinology, Women's Hospital, University of Heidelberg, Heidelberg, F.R.G.

The influence of environmental pollution on human reproductive performance is unknown. The toxicity of organochlorine compounds (OC) was recongized following accidental poisoning. In animal studies $O C$ administration resulted in a reduced number of pregnancies, and increased number of stillbirths. In the subsequent generation reduced fertility and chances in the menstrual cycle were noted. Wood preservatives containing various toxic $\mathrm{OC}$ are frequently used in industrialized states. Therefore it was the aim of this study to measure the pesticides pentachlorophenol (PCP) and gammahexachlorocyclohexane (Lindane, L) in women with hormonal disorders. In 22 out of 90 women examined elevated OC concentrations were found by capillary chromatography and masspectroscopy. PCP serum levels above $25 \mu \mathrm{g} / \mathrm{l}$ were judged elevated, as were L blood levels above $100 \mathrm{ng} / \mathrm{l}$. Elevated concentrations were found in 8 fertile women (6 of them with repeated abortions, aged 26-33 years), 8 women (aged 16 - 35 years) with menstrual disorders and androgenisation symptoms, and 6 women (aged 42 - 56 years) with climacteric symptoms. The source of emmission was identified in the residence of each patient, the duration of exposure ranged from 2 to 20 years. 16 women presented with adrenocortical malfunction ( 7 with hyperandrogenemia and 9 with partial adrenocorticoinsufficiency), 15 with immunological disorders, 6 with thyroid gland dysfunction (1 with hyper- and 5 with hypothyroidism) and 3 with luteal phase insufficiency. After elemination of the causative noxae and immunologic stimulation, blood $\mathrm{OC}$ concentrations decreased and the patients' physical conditions improved. menstruation normalized and, in case of conception, pregnancies resulted in healthy babies. On the basis of our data OC influence various hormonal systems including the adrenal and thyroid glands and reduce cellular immunity. Therefore an exact evaluation of environmental pollutions should be included in the endocrinological work-up of the patients with hormonal disorders and reproductive failure.

140. Atrial natriuretic peptide (ANP) and progesterone in ovarian follicluar fluid

E.A.P. Steegers, M. van Heeswijk, J.M.G. Hollanders, H.W. Jongsma, and P.R. Hein

Department of Obstetrics and Gynaecology, University Hospital Nijmegen, The Netherlands 